\title{
Spin State Ordering in Metal-Based Compounds Using the Localized Active Space Self-Consistent Field Method
}

\author{
Riddhish Pandharkar, Matthew R. Hermes, Christopher J. Cramer, Laura Gagliardi
}

Submitted date: 19/07/2019 - Posted date: 22/07/2019

Licence: CC BY-NC-ND 4.0

Citation information: Pandharkar, Riddhish; Hermes, Matthew R.; Cramer, Christopher J.; Gagliardi, Laura (2019): Spin State Ordering in Metal-Based Compounds Using the Localized Active Space Self-Consistent Field Method. ChemRxiv. Preprint.

Quantitatively accurate calculations for spin state ordering in transition-metal complexes typically demand a robust multiconfigurational treatment. The poor scaling of such methods with increasing size makes them impractical for large, strongly correlated systems. Density matrix embedding theory (DMET) is a fragmentation approach that can be used to specifically address this challenge. The single-determinantal bath framework of DMET is applicable in many situations, but it has been shown to perform poorly for molecules characterized by strong correlation when a multiconfigurational self-consistent field solver is used. To ameliorate this problem, the localized active space self-consistent field (LASSCF) method was recently described. In this work, LASSCF is applied to predict spin state energetics in mono- and di-iron systems and we show that the model offers an accuracy equivalent to CASSCF but at a substantially lower computational cost. Performance as a function of basis set and active space is also examined.

File list (2) 


\title{
Spin state ordering in metal-based compounds using
}

\section{the localized active space self-consistent field method}

\author{
Riddhish Pandharkar, Matthew R. Hermes, Christopher J. Cramer, and Laura \\ Gagliardi* \\ Department of Chemistry, Chemical Theory Center, and The Minnesota Supercomputing \\ Institute, University of Minnesota, Minneapolis, MN, 55455
}

Corresponding author: gagliard@umn.edu

\begin{abstract}
:
Quantitatively accurate calculations for spin state ordering in transition-metal complexes typically demand a robust multiconfigurational treatment. The poor scaling of such methods with increasing size makes them impractical for large, strongly correlated systems. Density matrix embedding theory (DMET) is a fragmentation approach that can be used to specifically address this challenge. The single-determinantal bath framework of DMET is applicable in many situations, but it has been shown to perform poorly for molecules characterized by strong correlation when a multiconfigurational self-consistent field solver is used. To ameliorate this problem, the localized active space self-consistent field (LASSCF) method was recently described. In this work, LASSCF is applied to predict spin state energetics in mono- and di-iron systems and we show that the model offers an accuracy equivalent to CASSCF but at a substantially lower computational cost. Performance as a function of basis set and active space is also examined.
\end{abstract}


TOC Graphic:

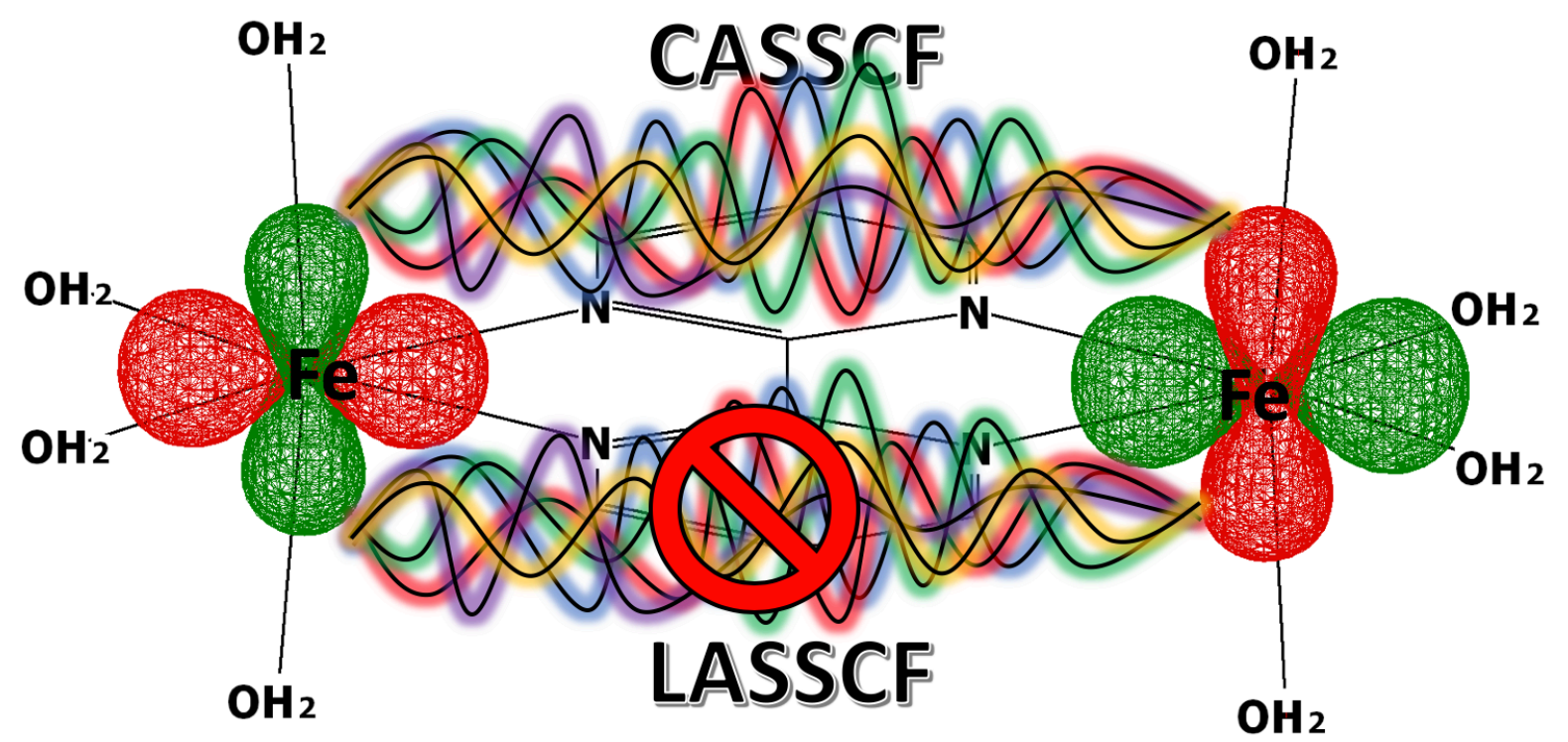

Increasingly efficient treatments of strongly correlated chemical systems has been a long-standing goal for electronic structure theory. Density functional theory is now widely used for many applications owing to its economical scaling and flexibility. ${ }^{1-4}$ However, in many instances, the accuracy offered by DFT is inadequate, and a multiconfigurational model is required. ${ }^{5}$ The calculations of spin-state energy ladders for many transition metal complexes present significant challenges. ${ }^{6,7}$ As crossing from one spin-state to another may be facile and have a significant effect on chemical reactivity, knowledge of the relative energies of different spin states for a given complex is essential to understand (and improve) its catalytic and magnetic properties. ${ }^{8,9}$ However, the poor scaling of most multiconfigurational methods makes them impractical for large, strongly correlated systems, especially ones having multiple metal centers. Although the variants of the complete active space self-consistent field (CASSCF) namely the restricted (RASSCF) $)^{10,11}$ and 
generalized (GASSCF) ${ }^{12}$ active space approaches can help reduce the cost, they too ultimately face limitations owing to their scaling. To obtain an acceptable multireference wave function for an arbitrarily large system, one needs to eliminate the exponential scaling with the size of the active space.

In many systems, the low-lying virtual orbitals that contribute the most to the multi-reference character are well localized, which makes fragmentation and embedding methods potentially efficient. ${ }^{13}$ However, a good fragmentation method must account for quantum interactions (electron correlation) between fragments.

Density matrix embedding theory is a wave function-in-wave function approach that allows fragmentation of strongly correlated systems. ${ }^{14}$ It is founded on the Schmidt decomposition of a wave function that identifies the degrees of freedom over which the fragment and its environment are entangled, and reduces the size of the problem to at most twice that of the fragment. Although the single-determinantal bath framework of DMET is applicable for simple problems, it performs poorly for molecules with strong correlation when a multi-configurational self-consistent field (MCSCF) solver is used for at least one fragments. ${ }^{15}$ To address this issue, a new type of MCSCF wave function called LAS (localized active space) SCF was proposed.${ }^{16}$ LASSCF reproduces CASSCF results for bond breaking in model test systems such as azomethane and bisdiazenes. ${ }^{16}$

We apply LASSCF to a mono- and a di-iron complex and to iron porphyrin and explore the performance of DMET and LASSCF for different basis sets and active spaces by calculating lowspin/high-spin energy splitting. We show that only the single embedding (i.e., a calculation with only one fragment) scheme of DMET shows reasonable agreement with a corresponding CASSCF treatment, and that LASSCF outperforms it in every case. We further demonstrate that with LASSCF one can obtain CASSCF quality results at a significantly reduced computational cost. 
In the four embedding schemes we compare, the user chooses a set of orbitals which define the fragments at the start of the calculation. For these fragment orbitals, a set of bath orbitals is constructed that models the effect of the rest of the molecule. The number of bath orbitals is no more than the number of fragment orbitals. The methods used to construct each bath are different in the four schemes. The space spanned by the fragment orbitals and their respective bath orbitals is called the embedding space or impurity space. A high-level theory (here CASSCF) solver is used in this embedding space.

First, we consider the single embedding scheme (DMET_1E) that treats only one fragment at a high-level of theory and the whole system at a lower level (here RHF) to give a wave function denoted by $\mid$ DMET_1E $\rangle$. The Hamiltonian in the embedding space for the $I^{\text {th }}$ fragment, $\widehat{H}_{I}$, is constructed by using a projection operator, $\hat{P}_{I}$,

$$
\begin{gathered}
\hat{P}_{I}=\sum_{\vec{f}, \vec{b}}^{N_{F_{k}}}|\vec{f}\rangle \otimes|\vec{b}\rangle\langle\vec{b}| \otimes\langle\vec{f}| \\
\widehat{H}_{I}=\hat{P}_{I} \widehat{H} \hat{P}_{I}
\end{gathered}
$$

Where $\vec{f}=\left\{f_{1}, f_{2}, \ldots\right\}$ and $\vec{b}=\left\{b_{1}, b_{2}, \ldots\right\}$ respectively list occupied fragment and bath orbitals in a single determinant of the fragment and bath Fock spaces. The bath orbitals are obtained by a Schmidt decomposition of the RHF wave function. The CASSCF solver gives a wave function, $\psi_{I}$, expressed in the basis of fragment and bath orbitals, that minimizes the energy for this embedding Hamiltonian, $\left\langle\psi_{I}\left|\hat{P}_{I} \widehat{H} \hat{P}_{I}\right| \psi_{I}\right\rangle$. The $\left|D M E T \_1 E\right\rangle$ is obtained as $\left|\Psi_{I}\right\rangle \otimes\left|\Phi_{c}\right\rangle$ , the product of this $\psi_{I}$ and a single determinant in the space of unentangled "core" orbitals which are neither fragment nor bath and whose occupations are given by the RHF wave function. 
The energy is the expectation value of the full molecule Hamiltonian $\widehat{H}$ for this $\mid$ DMET_1E $\rangle$ wave function

$$
E_{D M E T \_1 E}=\langle\text { DMET_1E }|\widehat{H}| \text { DMET_1E }\rangle
$$

$\mid$ DMET_1E $\rangle$ is an approximation to the $\mid$ CASSCF $\rangle$ wave function because active orbitals are confined to rotate only within the impurity orbitals, unlike conventional CASSCF. Thus $E_{D M E T-1 E}$ is an upper bound to the CASSCF energy.

This approach, however, it is not useful when multiple fragments require the higher level of theory. Here a multiple embedding scheme (DMET) is needed, where a different embedding Hamiltonian is created for each of the $N_{k}$ fragments in the system, and individual fragment solutions are then obtained separately. In this scheme the total energy is

$$
E_{D M E T}=V_{o}+\sum_{k}^{N_{k}}\left[h_{i_{k 1}}^{f_{k 1}} D_{i_{k 1}}^{f_{k 1}}+g_{i_{k 1} i_{k 3}}^{f_{k 1} i_{k 2}} d_{i_{k 2} i_{k 3}}^{f_{k 1} i_{k 2}}\right]
$$

where $V_{o}$ is the external potential energy, $h$ and $g$ are the one- and two-electron Hamiltonian integrals and $D$ and $d$ are the elements of the spin-summed one- and two-electron density matrices for the $k^{\text {th }}$ impurity wave function, and $f_{k n}\left(i_{k n}\right)$ indexes the $k^{\text {th }}$ set of fragment (fragment and bath) orbitals. The method is no longer variational and the total energy, $E_{D M E T}$, is not an upper bound to the corresponding CASSCF calculations.

The third scheme, self-consistent DMET (scDMET), is an extension of DMET and involves the optimization of a correlation potential that iteratively improves the bath. ${ }^{17}$

We have observed that the DMET and scDMET do not perform well for highly multiconfigurational molecules when a MCSCF impurity solver is used. ${ }^{15}$ The single determinantal bath, although extremely practical, fails when the active orbitals of one fragment are 
entangled with another fragment and thus become a part of the latter's bath. To address this problem, some of us developed a new method called LASSCF. ${ }^{16}$

When there are multiple fragments with strong correlation internally but weak correlation between them, the active space can be treated as several non-overlapping, mutually unentangled subspaces localized on the various fragments. The part of the total active space $(A)$ belonging to the $k^{\text {th }}$ fragment is referred to as $A_{k}$. This localized active space (LAS) wave function is an antisymmetric product of all the active sub-space wave functions and an inactive determinant,

$$
|L A S\rangle=\left[\otimes_{k}\left|\psi_{A_{k}}\right\rangle\right] \otimes\left|\Phi_{U}\right\rangle
$$

This expression of the wave function is not restricted to the context of fragmentation methods. Previous methods have established that such fragmentations are helpful in many cases. ${ }^{18-21}$ The standard DMET framework, however, is convenient for variationally optimizing the LAS wave function. An impurity problem is constructed for each fragment with bath orbitals obtained from the occupied inactive orbitals of $\left|\Phi_{U}\right\rangle$. Details of the initialization are given in the Supporting Information. A CASSCF solver is used to obtain a wave function (the active orbitals and CI vectors) within the fragment. When taken as a product, these localized active space wave functions give the LAS wave function for the entire system. Like single-embedding DMET, the LASSCF energy

$$
E_{\mathrm{LAS}}=\langle\mathrm{LAS}|\widehat{H}| \mathrm{LAS}\rangle
$$

is variational and an upper bound to the comparable CASSCF energy. ${ }^{16}$

An important characteristic of the LASSCF calculations reported in this work is that the LAS wave function is a product of local wave functions that are not themselves all closed-shell singlets. The energy formula for LASSCF has to account for this. The expansion of the projected Hamiltonian 
of the $k^{\text {th }}$ fragment, $\widehat{H}_{I_{k}} \equiv \widehat{P}_{I_{k}} \widehat{H} \widehat{P}_{I_{k}}$ (where $\widehat{P}_{I_{k}}$ is defined as in equation 1$)$ includes the following term:

$$
\frac{1}{2} g_{a_{l 2} i_{k 2}}^{i_{k 1} a_{l 1}}\left(\gamma_{a_{l 2} \downarrow}^{a_{l 1} \downarrow}-\gamma_{a_{l 2} \uparrow}^{a_{l 1} \uparrow}\right)\left(\hat{c}_{i_{k 1} \uparrow}^{\dagger} \hat{c}_{i_{k 2} \uparrow}-\hat{c}_{i_{k 1} \downarrow}^{\dagger} \hat{c}_{i_{k 2} \downarrow}\right)
$$

where $a_{l 1}$ indexes the active orbitals of the $l^{\text {th }}$ fragment $(k \neq l), \gamma$ is the density matrix element in the spin-orbital basis, $g_{a_{l 2} i_{k 2}}^{i_{k 1} a_{l 1}}$ is the two-electron Hamiltonian integral and $\hat{c}^{\dagger} \hat{c}$ are the creation and annihilation operators for both electrons with spins alpha $(\uparrow)$ and beta $(\downarrow)$.

The value of the spin density matrix, $\left(\gamma_{a_{l 2} \downarrow}^{a_{l 1} \downarrow}-\gamma_{a_{l 2} \uparrow}^{a_{l 1} \uparrow}\right)$, is zero when all electrons outside of the $k^{t h}$ fragment are singlet-coupled. If, however, multiple fragments have non-singlet spins, this term is not identically zero. In such cases, the LAS wave function will not be an eigenstate of the total spin operator, viz.:

$$
\begin{aligned}
\left\langle L A S\left|\hat{S}^{2}\right| L A S\right\rangle & =\sum_{k}\left\langle\psi_{A_{k}}\left|\hat{S}^{2}\right| \psi_{A_{k}}\right\rangle \\
& =\sum_{k} s_{k}\left(s_{k}+1\right)|L A S\rangle \neq s(s+1)|L A S\rangle
\end{aligned}
$$

where $s_{k}$ is the spin for the $k^{\text {th }}$ fragment and $s$ is the whole-system total spin quantum number.

All calculations were performed using the 'mrh' code available in github ${ }^{22}$ which is based on QC$\mathrm{DMET}^{23}$ and invokes PySCF${ }^{24}$. We studied three molecules: $\left[\mathrm{FeNCH}_{6}\right]^{+2}, \mathrm{Fe}$-porphyrin and $\left[\mathrm{Fe}\left(\mathrm{H}_{2} \mathrm{O}\right)_{4}\right]$ bpym $^{+4}$ (bpym= 2,2'-bipyrimidine), hereafter referred to as systems $\mathbf{1}$, 2, and $\mathbf{3}$ respectively (Figure 1). Details of geometry optimizations, fragmentation, and active space selection are reported in the Supporting Information. 


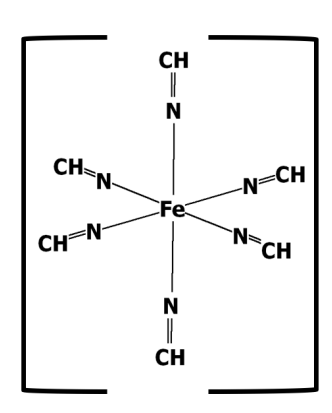

1
II

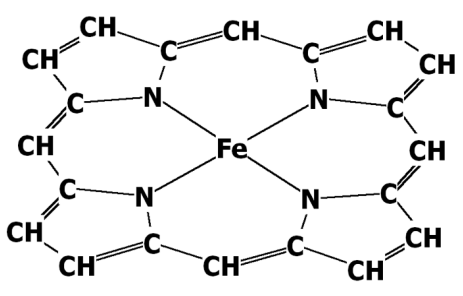

2

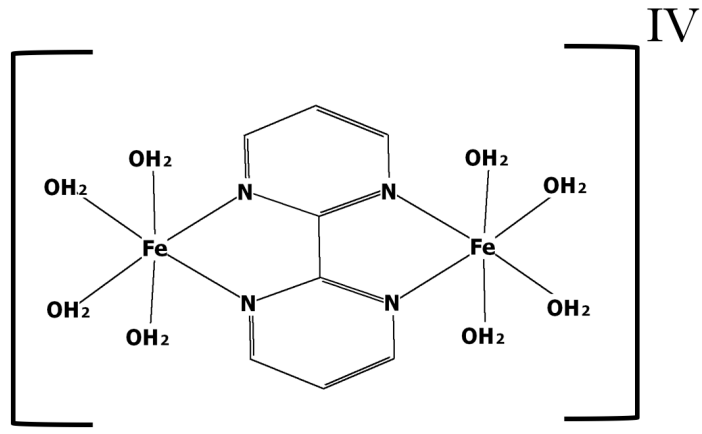

3

Figure 1: The molecules studied: System 1- $\left[\mathrm{Fe}(\mathrm{NCH})_{6}\right]^{+2}$ (left) is an octahedral complex, System 2 - Iron-porphyrin (center) is a square planar structure and System $3-\left[\mathrm{Fe}\left(\mathrm{H}_{2} \mathrm{O}\right)_{4}\right] \mathrm{bpym}^{+4}$ is a bimetallic complex.

Table 1: The quintet- singlet gaps in kcal/mol for system 1 calculated with various basis sets and active spaces for the methods being compared.

\begin{tabular}{|c|c|c|c|c|c|}
\hline Basis Set & \multicolumn{2}{|c|}{ 6-31G } & \multicolumn{3}{c|}{ 6-31G* } \\
\hline Active Space & $\mathbf{( 6 , 5 )}$ & $\mathbf{( 6 , 1 0 )}$ & $\mathbf{( 6 , 5 )}$ & $\mathbf{( 6 , 1 0 )}$ & $\mathbf{( 1 2 , 1 4 )}$ \\
\hline CASSCF & -77.9 & -67.6 & -72.8 & -62.1 & -47.2 \\
\hline DMET_1E & -75.4 & -64.9 & -70.1 & -59.6 & -45.4 \\
\hline DMET & -180.6 & - & - & - & - \\
\hline ScDMET & 90.3 & - & - & - & - \\
\hline LASSCF & -78.2 & -67.5 & -73.1 & -62.7 & -48.3 \\
\hline
\end{tabular}

Quintet-singlet gaps for 1 with the various embedding schemes and CASSCF are presented in Table 1 for different active spaces and basis sets. The DMET_1E embedding scheme reproduces the CASSCF reference gap well (absolute energies are also reproduced well, see Table S1 of SI). On the other hand, the gap predicted by multiple embedding schemes (DMET and scDMET) are very different from the reference. For this reason, these two schemes were not tested for any other calculation. LASSCF outperforms DMET_1E and differ from CASSCF by an average of less than $0.6 \mathrm{kcal} / \mathrm{mol}$ over the various comparisons (See SI Tables S1 and S2 of SI). 
LASSCF outperforms DMET and scDMET because of the superior self-consistency in the former between the total energy expression and the model of entanglement used to construct bath orbitals. In DMET, some of the Fe $3 d$ (active) orbitals contribute to the bath of the ligand fragments (Figure 3). This derives from the unphysical RHF description of the electrons occupying these $3 d$ orbitals which, in the more accurate CASSCF and LASSCF wave functions, are not entangled to any of the inactive or virtual orbitals and so should not appear in any fragment's bath space. Since, in the multi-fragment DMET and scDMET cases, we also use RHF to compute the impurity wave function of the ligand, the RHF picture of the $3 d$ orbitals also generates an unphysical contribution to the total DMET energy [Eq (4)]. The equivalent embedding orbitals constructed for the ligands in the LASSCF method have none of the Fe 3d (active) orbitals of the iron.

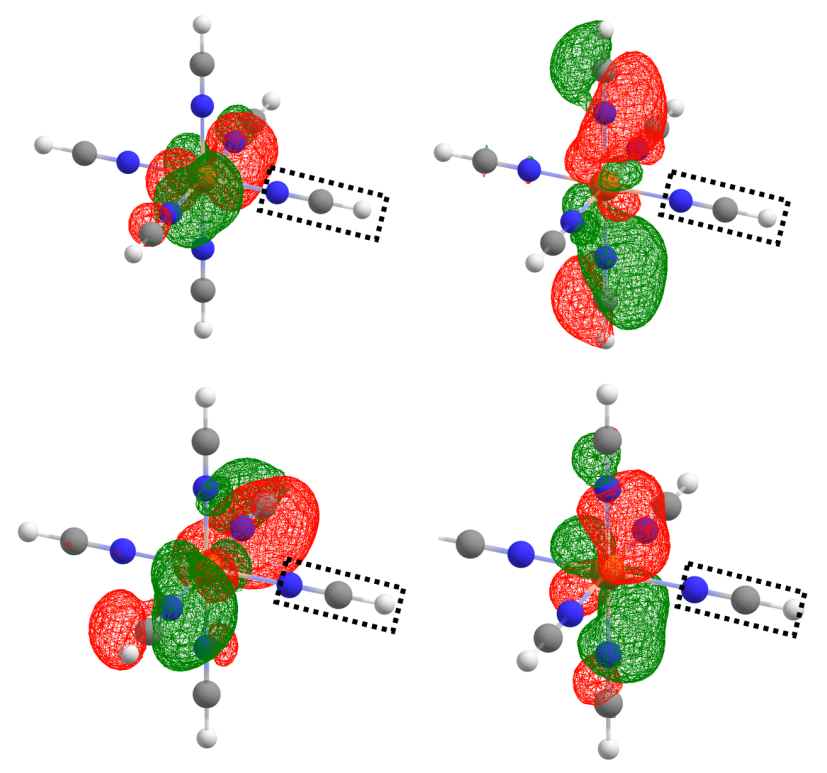

Figure 2: Some of the impurity orbitals for the ligand fragment (in the dotted box) of a singlet calculation in system 1. Some of the orbitals in the bath constructed for the ligand include active orbitals of the iron atom.

Embedding methods are sensitive to the choice of basis set. ${ }^{25}$ Table 2 explores the performance of DMET_1E and LASSCF with three different active spaces and the 6-31G and 6-31G* bases. ${ }^{26,27}$ 
Table 2 : DMET_IE and LASSCF energy differences ( $k c a l / m o l)$ with respect to the CASSCF energy for $\mathrm{Fe}(\mathrm{NCH})_{6}$ calculated using the 6-31G and 6-31G* basis sets and $(6,5),(6,10)$ and $(12,14)$ active spaces

\begin{tabular}{|c|c|c|c|c|}
\hline Basis Set & Active Space & Spin State & DMET_1E & LASSCF \\
\hline \multirow{3}{*}{$6-31 \mathrm{G}$} & \multirow{2}{*}{$(6,5)$} & Singlet & 0.3 & 0.2 \\
\cline { 3 - 5 } & \multirow{2}{*}{$(6,10)$} & Quintet & 2.8 & 0.0 \\
\cline { 3 - 5 } & \multirow{2}{*}{$(6,5)$} & Singlet & 1.2 & 0.1 \\
\cline { 3 - 5 } & & Quintet & 3.9 & 0.3 \\
\cline { 3 - 5 } & & Singlet & 0.3 & 0.2 \\
\cline { 3 - 5 } & \multirow{4}{*}{$6-31 \mathrm{G}^{*}$} & Quintet & 3.0 & 0.0 \\
\cline { 3 - 5 } & \multirow{2}{*}{$(6,10)$} & Singlet & 1.9 & 1.8 \\
\cline { 3 - 5 } & \multirow{2}{*}{$(12,14)$} & Quintet & 4.4 & 1.1 \\
\cline { 3 - 5 } & & Singlet & 2.9 & 2.4 \\
\cline { 3 - 5 } & Quintet & 4.7 & 1.4 \\
\hline
\end{tabular}

The difference with respect to CASSCF increases with the basis set size for both DMET_1E and LASSCF. The relative paucity of correlating virtual orbitals in the bath causes the embedding space calculations to have fewer degrees of freedom than the CASSCF calculation. This difference increases with increasing basis size. Table 2 also shows dependence on the active space size. As increasingly diffuse orbitals are added to the iron atom's active space, the paucity of diffuse virtual orbitals in the iron impurity subspace imposes an increased variational penalty on both DMET_1E and LASSCF. However, even in the case of the largest basis set, the error of the singlet-quintet gap is dominated by the choice different active spaces.

Table 3 shows energies relative to CASSCF for system 2 for DMET_1E and LASSCF. The coordination environment for the iron atom is different compared to system $\mathbf{1}$ and the environment includes significantly more basis functions than system 1. Here, too, LASSCF significantly outperforms DMET_1E. 
Table 3: The energies $(\mathrm{kcal} / \mathrm{mol})$ relative to the CASSCF calculations for system 2 calculated using $6-31 G^{*}$ basis set and $(6,5)$ and $(6,10)$ active spaces

\begin{tabular}{|c|c|c|c|}
\hline Active Space & Spin State & DMET_1E & LASSCF \\
\hline \multirow{2}{*}{$(6,5)$} & Singlet & 0.3 & 0.2 \\
\cline { 2 - 4 } & Quintet & 17.0 & 1.3 \\
\hline \multirow{2}{*}{$(6,10)$} & Singlet & 0.8 & 0.5 \\
\cline { 2 - 4 } & Quintet & 6.7 & 3.5 \\
\hline
\end{tabular}

For system 1, the $(6,5)$ LASSCF and CASSCF quintet energies are equal because we use a restricted open shell HF solution as the initial guess for the LASSCF calculation and for system $\mathbf{1}$, with a $(6,5)$ active space of the $3 \mathrm{~d}$ iron orbitals, this is the identical to the CASSCF solution. This is not the case for system $\mathbf{2}$, however, where the singly occupied ROHF orbitals are not all $3 d$ orbitals. In particular, as shown in Figure 3, four singly occupied orbitals are localized on the porphyrin molecule, not the iron atom, and that is why the error for the LASSCF quintet is not zero. 

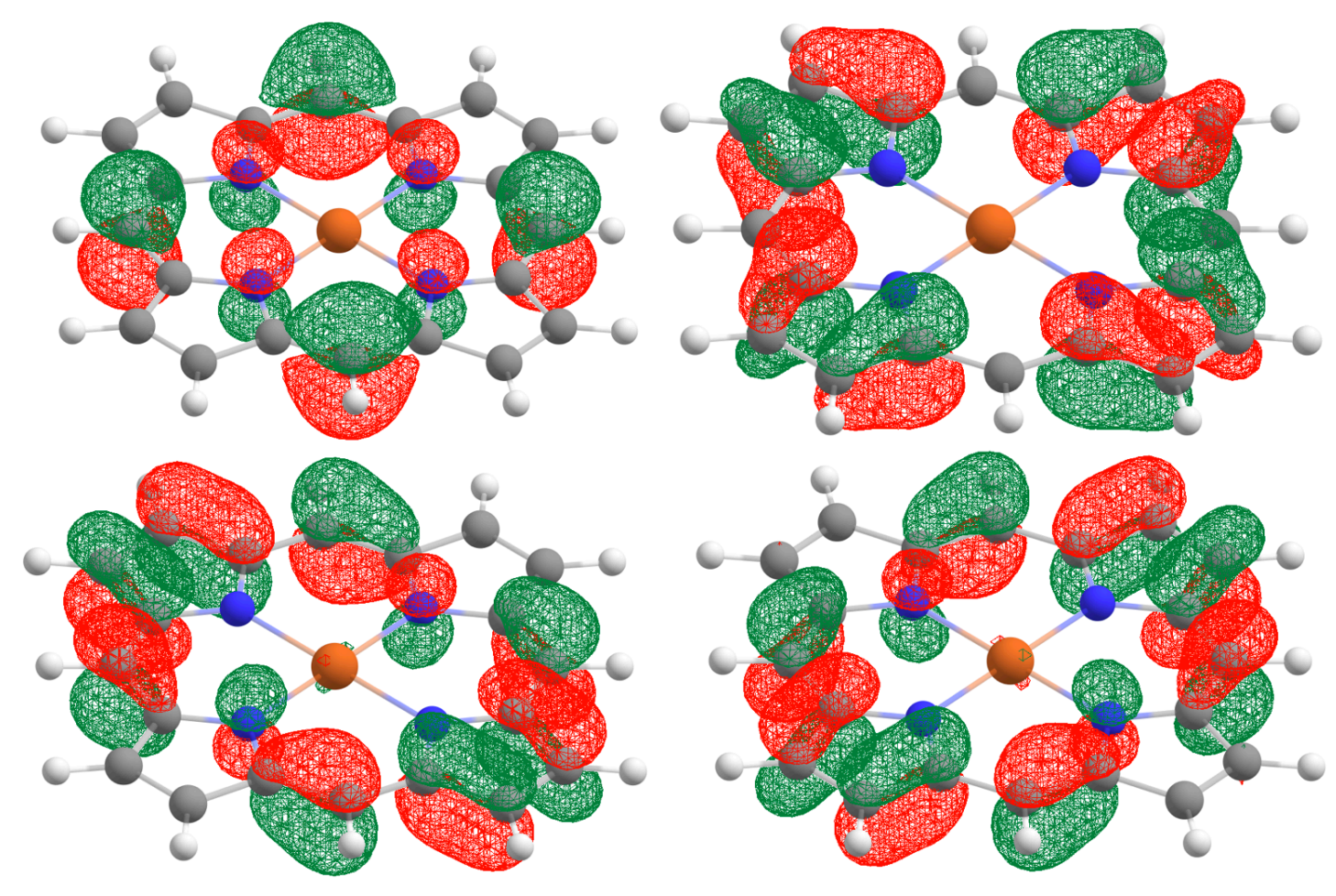

Figure 3: Singly occupied ROHF orbitals for the quintet in a 6-31G basis. Unlike for system 1, these are not the active orbitals in the (6.5) case.

System 3 has an antiferromagnetic coupling between two locally high-spin Fe(II) atoms. ${ }^{28}$ This belongs to a family of molecules whose spin interactions have been extensively studied in the literature. ${ }^{29-31}$ The physical separation of the two iron centers motivates us to treat them as two different fragments, with a $(6,5)$ active space on each. The resulting LAS wave function includes vastly fewer configurations than the corresponding CAS wave function, thereby reducing the computational cost (Table 4).

We consider the overall singlet and nonet states. While for CASSCF we simply impose the total spin, in LASSCF we need to specify the spin on each fragment. This is straightforward for the nonet where both iron centers have a local quantum number $m_{s}$ equal to 2 . For the overall singlet state, the CASSCF solution is a linear combination of the diamagnetic singlets and anti- 
ferromagnetically coupled triplets and quintets. In LASSCF however, we can consider only one such case at a time; namely the diamagnetic singlet on both centers and antiferromagnetically coupled triplets (i.e., $m_{s} @ \mathrm{Fe} 1=+1$ and $m_{s} @ \mathrm{Fe} 2=-1$ ) and quintets (i.e., $m_{s} @ \mathrm{Fe} 1=+2$ and $m_{s}$ (a) $\mathrm{Fe} 2=-2$ ). Energies relative to the CASSCF nonet are shown in table 4. All calculations were performed at the same nonet optimized geometry. The absolute energies for these calculations are reported in the table S5 in the SI. The CASSCF singlet-nonet gap is $0.0 \mathrm{kcal} / \mathrm{mol}$ indicating that there is a degeneracy (or near degeneracy) in the nonet and a (possibly broken symmetry) singlet wave function. The LASSCF nonet is only $1.5 \mathrm{kcal} / \mathrm{mol}$ higher than the CASSCF nonet. The LASSCF singlet calculated using the antiferromagnetically coupled quintets is the most stable of the couplings considered while the diamagnetic singlet is the least stable. LASSCF also successfully captures the degeneracy between a broken symmetry singlet and the nonet wave function.

Table 4: The energies $(\mathrm{kcal} / \mathrm{mol})$ relative to the CASSCF nonet for system 3 calculated using 6$31 G^{*}$ basis set and a $(6,5)$ active space on each iron for LASSCF and $(12,10)$ active space for the CASSCF together with the number of Slater Determinants (SDs) required to span the space of all configurations considered.

\begin{tabular}{|c|c|c|c|c|c|c|}
\hline $\begin{array}{c}\text { Overall } \\
\text { Spin }\end{array}$ & E $_{\text {CASSCF }}$ & $\begin{array}{c}\text { SDs for } \\
\text { CASSCF }\end{array}$ & $\begin{array}{c}\mathbf{m}_{\mathbf{s}} \text { for Fe1 } \\
(\mathbf{L A S})\end{array}$ & $\begin{array}{c}\mathbf{m}_{\mathbf{s}} \text { for Fe2 } \\
\text { (LAS) }\end{array}$ & $\mathbf{E}_{\text {LASSCF }}$ & $\begin{array}{c}\text { SDs for } \\
\text { LASSCF }\end{array}$ \\
\hline & & & 0 & 0 & 129.5 & 200 \\
\cline { 4 - 7 } & & & 1 & -1 & 99.4 & 100 \\
\cline { 4 - 7 } Singlet & 0.0 & 44100 & 2 & -2 & 1.5 & 10 \\
\hline Nonet & 0.0 & 45 & 2 & 2 & 1.5 & 10 \\
\hline
\end{tabular}

But the analysis also gives us more insight in behavior of the spin interactions. The control LASSCF provides over the spin in each fragment is useful to dissect the wave function of the overall singlet into its contributing couplings and get an idea of their individual contribution without having obtained the overall singlet CASSCF wave function. 
Even though the LAS wave function is not a proper spin eigenfunction when multiple fragments have non-singlet spins, because each active subspace part of the LAS wave function is individually spin-adapted with both total $\left(s_{K}\right)$ and projection $\left(m_{K}\right)$ spin quantum numbers, it is straightforward in principle to generate spin-adapted linear combinations of LAS states utilizing Clebsch-Gordan coefficients. ${ }^{32}$ These states are sums over products of all states with $-s_{K} \leq m_{K} \leq s_{K}$ for the $K^{\text {th }}$ fragment consistent with a fixed set of fragment total spin quantum numbers $\left(-s_{K}\right)$, a given whole-system total spin quantum number $(s)$, and a given whole-system spin projection quantum number $\left(m=\Sigma_{K} m_{K}\right)$. The procedure is a generalization of the procedure for generating spin-adapted configuration state functions (CSFs) as linear combinations of single determinants that lack a well-defined total spin quantum number but which have a spin projection quantum number, and which are product states over subsystems (individual electrons) which have both quantum numbers well-defined.

Concluding Remarks: The LASSCF method is an effective way of treating molecules with locally strong electron correlation in the context of embedding methods. We have for the first time demonstrated the performance of this method for spin-state calculations in iron-coordination compounds and tested the performance of the method with various active spaces and basis sets. We compared the method to three variants of CAS-DMET to highlight the importance of unentangling the active subspaces from each other and the rest of the molecule. Using a bimetallic complex, we show that LASSCF can be used in systems with coupled, locally high-spin centers. LASSCF is especially computationally appealing as the number of spin centers grows because the cost of the calculation scales only linearly after accounting for the size of the active space(s) of the individual fragments and the overall wave function is a product of localized wave functions. We 
anticipate that LASSCF will serve as a good reference wave function when additional correlation energy is computed using methods like perturbation theory or pair-density functional theory.

Supporting Information: The computational details, absolute energies for all calculations (CASSCF, DMET and LASSCF) and optimized geometries and energies for all compounds and each spin states are reported in the supporting information.

Acknowledgement: We thank Sina Chiniforoush, Hung Quam Pham and Donald G. Truhlar for useful discussion. L.G. had useful discussion with Karin Fink and Christoph van Wüllen. This research is supported by the U.S. Department of Energy, Office of Basic Energy Sciences, Division of Chemical Sciences, Geosciences and Biosciences under Award DEFG02-17ER16362. Computer resources were provided by the Minnesota Supercomputing Institute at the University of Minnesota.

References:

(1) Kohn, W.; Sham, L. J. Self-Consistent Equations Including Exchange and Correlation Effects. Phys. Rev. 1965, 140 (4A), A1133.

(2) Becke, A. D. Density-Functional Thermochemistry. III. The Role of Exact Exchange. $J$. Chem. Phys. 1993, 98 (7), 5648-5652.

(3) Lee, C.; Yang, W.; Parr, R. G. Development of the Colle-Salvetti Correlation-Energy Formula into a Functional of the Electron Density. Phys. Rev. B 1988, 37 (2), 785.

(4) Zhao, Y.; Truhlar, D. G. The M06 Suite of Density Functionals for Main Group Thermochemistry, Thermochemical Kinetics, Noncovalent Interactions, Excited States, and Transition Elements: Two New Functionals and Systematic Testing of Four M06Class Functionals and 12 Other Function. Theor. Chem. Acc. 2008, 120 (1-3), 215-241.

(5) Adamo, C.; Barone, V. Toward Reliable Density Functional Methods without Adjustable Parameters: The PBE0 Model. J. Chem. Phys. 1999, 110 (13), 6158-6170. https://doi.org/10.1063/1.478522.

(6) Ioannidis, E. I.; Kulik, H. J. Ligand-Field-Dependent Behavior of Meta-GGA Exchange in 
Transition-Metal Complex Spin-State Ordering. J. Phys. Chem. A 2017, 121 (4), 874-884. https://doi.org/10.1021/acs.jpca.6b11930.

(7) Ioannidis, E. I.; Kulik, H. J. Towards Quantifying the Role of Exact Exchange in Predictions of Transition Metal Complex Properties. J. Chem. Phys. 2015, 143 (3), 034104. https://doi.org/10.1063/1.4926836.

(8) Harvey, J. N.; Poli, R.; Smith, K. M. Understanding the Reactivity of Transition Metal Complexes Involving Multiple Spin States. Coord. Chem. Rev. 2003, 238, 347-361.

(9) Halcrow, M. A. Structure: Function Relationships in Molecular Spin-Crossover Complexes. Chem. Soc. Rev. 2011, 40 (7), 4119-4142.

(10) Olsen, J.; Roos, B. O.; Jo/rgensen, P.; Jensen, H. J. A. Determinant Based Configuration Interaction Algorithms for Complete and Restricted Configuration Interaction Spaces. $J$. Chem. Phys. 1988, 89 (4), 2185-2192. https://doi.org/10.1063/1.455063.

(11) Malmqvist, P.-Å.; Rendell, A.; Roos, B. O. The Restricted Active Space Self-ConsistentFleld Method, Implemented with a Split Graph Unitary Group Approach; 1990; Vol. 94.

(12) Ma, D.; Li Manni, G.; Gagliardi, L. The Generalized Active Space Concept in Multiconfigurational Self-Consistent Field Methods. J. Chem. Phys. 2011, 135 (4), 044128. https://doi.org/10.1063/1.3611401.

(13) Wójcik, M. J.; Nakatsuji, H.; Kirtman, B.; Ozaki, Y. Frontiers of Quantum Chemistry; Springer, 2018.

(14) Knizia, G.; Chan, G. K.-L. Density Matrix Embedding: A Simple Alternative to Dynamical Mean-Field Theory. Phys. Rev. Lett. 2012, 109 (18), 186404.

(15) Pham, H. Q.; Bernales, V.; Gagliardi, L. Can Density Matrix Embedding Theory with the Complete Activate Space Self-Consistent Field Solver Describe Single and Double Bond Breaking in Molecular Systems? J. Chem. Theory Comput. 2018, 14 (4), 1960-1968.

(16) Hermes, M. R.; Gagliardi, L. Multiconfigurational Self-Consistent Field Theory with Density Matrix Embedding: The Localized Active Space Self-Consistent Field Method. J. Chem. Theory Comput. 2019, 15 (2), 972-986. https://doi.org/10.1021/acs.jctc.8b01009. Wouters, S.; Jiménez-Hoyos, C. A.; Sun, Q.; Chan, G. K.-L. A Practical Guide to Density Matrix Embedding Theory in Quantum Chemistry. J. Chem. Theory Comput. 2016, 12 (6), 2706-2719.

(18) Parker, S. M.; Seideman, T.; Ratner, M. A.; Shiozaki, T. Communication: Active-Space Decomposition for Molecular Dimers. J. Chem. Phys. 2013, 139 (2), 021108. https://doi.org/10.1063/1.4813827.

(19) Parker, S. M.; Shiozaki, T. Communication: Active Space Decomposition with Multiple Sites: Density Matrix Renormalization Group Algorithm. J. Chem. Phys. 2014, 141 (21), 211102. https://doi.org/10.1063/1.4902991.

(20) Nakano, H.; Hirao, K. A Quasi-Complete Active Space Self-Consistent Field Method. Chem. Phys. Lett. 2000, 317 (1-2), 90-96. https://doi.org/10.1016/S0009-2614(99)013640 .

(21) Wu, W.; Su, P.; Shaik, S.; Hiberty, P. C. Classical Valence Bond Approach by Modern Methods. Chem. Rev. 2011, 111 (11), 7557-7593. https://doi.org/10.1021/cr100228r.

(22) Hermes, M. R. https://github.com/MatthewRHermes/mrh.

(23) Wouters, S. Https://Github.Com/Sebwouters/Qc-Dmet. 2016.

(24) Sun, Q.; Berkelbach, T. C.; Blunt, N. S.; Booth, G. H.; Guo, S.; Li, Z.; Liu, J.; McClain, J. D.; Sayfutyarova, E. R.; Sharma, S.; et al. PySCF: The Python-based Simulations of Chemistry Framework. Wiley Interdisciplinary Reviews: Computational Molecular 
Science. 2017, p e1340. https://doi.org/10.1002/wcms.1340.

(25) Wouters, S.; Jiménez-Hoyos, C. A.; Chan, G. K.-L. Five Years of Density Matrix Embedding Theory. arXiv Prepr. arXiv1605.05547 2016.

(26) Rassolov, V. A.; Pople, J. A.; Ratner, M. A.; Windus, T. L. 6-31G * Basis Set for Atoms K through Zn. J. Chem. Phys. 1998, 109 (4), 1223-1229. https://doi.org/10.1063/1.476673.

(27) Frisch, M. J.; Pople, J. A.; Binkley, J. S. Self-consistent Molecular Orbital Methods 25. Supplementary Functions for Gaussian Basis Sets. J. Chem. Phys. 1984, 80 (7), 32653269. https://doi.org/10.1063/1.447079.

(28) Gaspar, A. B.; Muñoz, M. C.; Real, J. A. Dinuclear Iron( Ii ) Spin Crossover Compounds: Singular Molecular Materials for Electronics. J. Mater. Chem. 2006, 16 (26), 2522-2533. https://doi.org/10.1039/B603488H.

(29) Moussa, N. O.; Trzop, E.; Mouri, S.; Zein, S.; Molnár, G.; Gaspar, A. B.; Collet, E.; Buron-Le Cointe, M.; Real, J. A.; Borshch, S.; et al. Wavelength Selective Light-Induced Magnetic Effects in the Binuclear Spin Crossover Compound \{ [ Fe ( Bt ) ( NCS ) 2 ] 2 (Bpym ) \}. Phys. Rev. B 2007, 75 (5), 054101. https://doi.org/10.1103/PhysRevB.75.054101.

(30) And, S. Z.; Borshch, S. A. Energetics of Binuclear Spin Transition Complexes. 2005. https://doi.org/10.1021/JA054282K.

(31) Wagner, S.; Kisslinger, F.; Ballmann, S.; Schramm, F.; Chandrasekar, R.; Bodenstein, T.; Fuhr, O.; Secker, D.; Fink, K.; Ruben, M.; et al. Switching of a Coupled Spin Pair in a Single-Molecule Junction. Nat. Nanotechnol. 2013, 8 (8), 575-579. https://doi.org/10.1038/nnano.2013.133.

(32) Helgaker, T.; Jorgensen, P.; Olsen, J. Molecular Electronic-Structure Theory; John Wiley \& Sons, 2014. 


\section{Supporting Information}

\section{Spin state ordering in metal-based compounds using the localized active space self-consistent field method}

Riddhish Pandharkar, Matthew R. Hermes, Christopher J. Cramer, and Laura Gagliardi*

Department of Chemistry, Chemical Theory Center, and The Minnesota Supercomputing Institute, University of Minnesota, Minneapolis, MN, 55455

Corresponding author: gagliard@umn.edu

Table of contents:

○ Computational details $\quad$ S2

- LASSCF calculations - technical aspects S3

- Absolute energies for CASSCF, DMET and LASSCF calculations S5

- Geometries optimized at the PBE/Def2TZVP level of theory $\quad$ S7 


\section{Computational Details}

Geometries for the singlet and quintet spin states for systems $\mathbf{1}$ and $\mathbf{2}$ and the nonet for system $\mathbf{3}$ were optimized using Gaussian $09 .{ }^{1}$ The PBE ${ }^{2}$ exchange correlation functional and a def2TZVP basis set ${ }^{3}$ was used. The geometries were optimized using ultrafine grids and without symmetry constraints. Vibrational frequency calculations were also done at the same level to ensure that the geometries correspond to true minima. While the respective geometries were used for each spin state for systems $\mathbf{1}$ and $\mathbf{2}$, the same geometry (nonet) was used for all CASSCF and LASSCF calculations for system 3.

For the mono metallic molecules, the iron atom is taken to be one fragment and is treated with CASSCF, while the rest of the molecule is taken to be either one fragment treated with RHF, or further divided in multiple fragments that are all treated at the RHF level. Three active spaces were studied for system 1; namely the $(6,5),(6,10)$ and $(12,14)$. The $(6,5)$ contains the five $3 d$ orbitals on the iron while the $(6,10)$ has the additional 3d' corelating orbitals. The $(12,14)$ active space has the $\sigma$, $\sigma$ ' (two Fe-ligand bonding orbitals) as well as the $3 \mathrm{~s}$ and $3 \mathrm{~s}$ ' orbitals in addition to those in the $(6,10)$ active space as shown in Figure S1. For system $\mathbf{2}$, only the $(6,5)$ and $(6,10)$ active spaces were studied. For system $\mathbf{3}$, the two iron atoms were considered as two separate fragments in the LASSCF calculation with each one having an active space of $(6,5)$ which was compared to the CASSCF calculation with a $(12,10)$ active space formed by the union of the $(6,5)$ active spaces on the two metals. The CASSCF and LASSCF calculations were performed using a 6-31G and 6-31G* basis for system 1 while only the 6-31G* basis was used for the other two systems. 


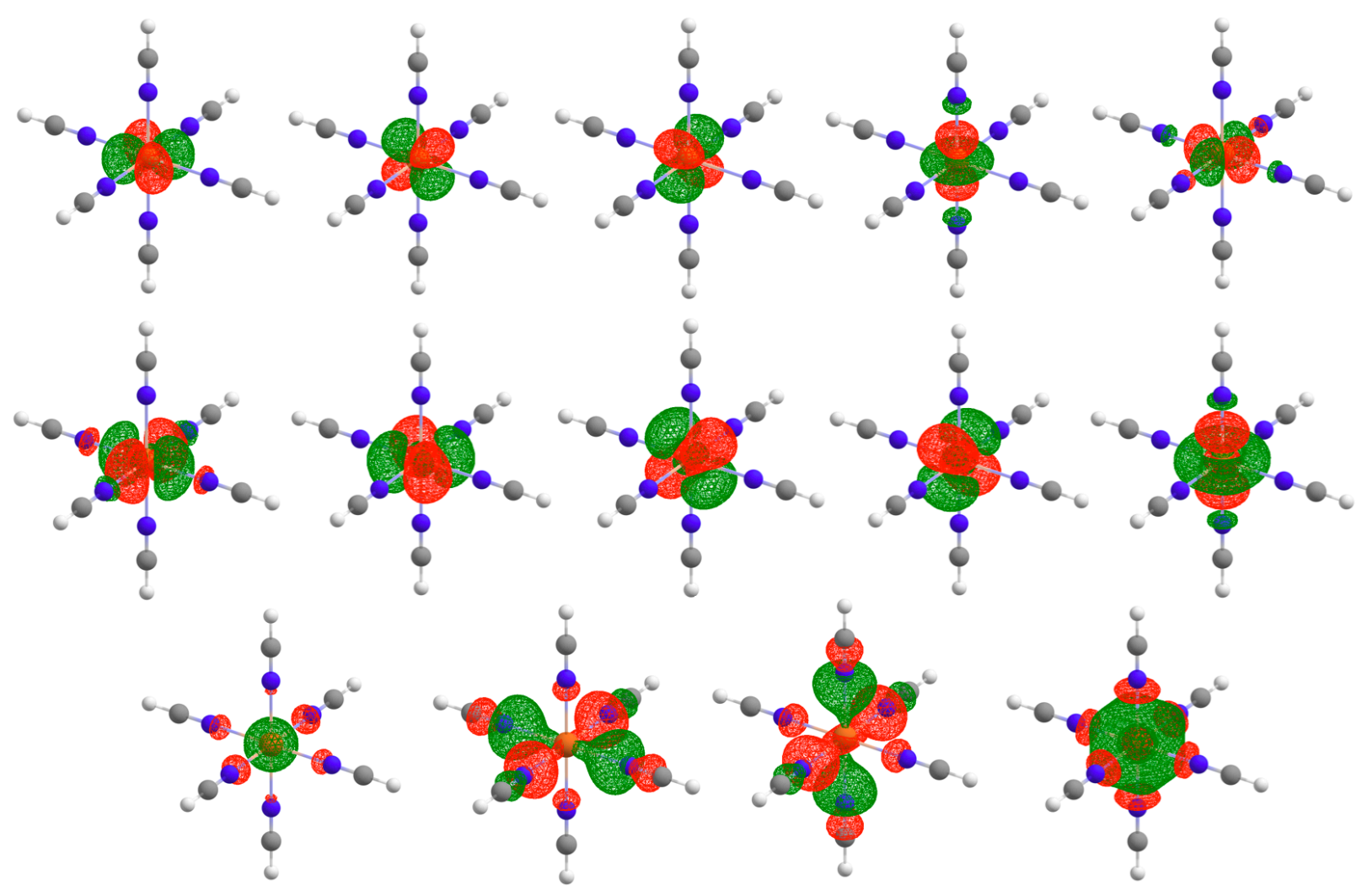

Figure S1: Active Orbitals corresponding to $(12,14)$ Active Space. The first two rows show the $(6,10)$ Active Space and the first row shows the (6,5) Active Space

\section{LASSCF calculations - technical aspects}

\section{A. Localization and fragment definitions:}

The RHF/ROHF solution serves as an initial guess for the calculation. Guess fragment orbitals are supplied by the user in way similar to DMET. A localization scheme, e.g. meta Löwdin, is used to generate a set of orthogonalized atomic orbitals. ${ }^{4,5}$ In further iterations, the definition of fragment orbitals changes but the guess fragment orbitals are not discarded and are used to ensure fragment orbitals in each iteration are localized. 


\section{B. Initialization and guess wave function generation:}

The RHF and ROHF orbitals generated by the initial calculation are also used for defining the active space for each fragment in the first iteration. The initial guess fragment orbitals are chosen to resemble as closely as possible the orthogonalized AOs of each fragment's constituent atom(s) under the constraint that they enclose the corresponding guess active orbitals. For the monometallic compounds the active orbitals were picked directly from the RHF/ROHF orbitals. For the bi-metallic system the active orbitals were constructed from a RHF (singlet) calculation using the atomic valence active space (AVAS) ${ }^{6}$ procedure and localized on the two separate iron atoms by a unitary transformation. The wave function for both open-shell states was initialized as a quadruply-excited Slater determinant - i.e. by changing the occupation numbers of 4 pairs of occupied and unoccupied orbitals in a closed shell RHF determinant.

\section{C. Defining properties of the fragments:}

Even though the user does not explicitly assign active orbitals to specific fragments, each fragment has the number of active orbitals and electrons defined. For instance, for a LASSCF calculation in system $\mathbf{3}$, we do define a $(6,5)$ active space for each fragment but we provide a list of 10 orbitals for the program to localize and then assign to either of the atoms. The user also defines the total number of unpaired electrons and the magnetization for each fragment. Similarly, the user also specifies the fragments to be treated with RHF. 


\section{Absolute Energies for CASSCF, DMET and LASSCF calculations.}

Table S1: Singlet and quintet energies (in Hartree) and gaps (in $\mathrm{kcal} / \mathrm{mol}$ ) for system 1 with a $(6,5)$ active space in a 6-31G basis using various DMET schemes and LASSCF.

\begin{tabular}{|c|c|c|c|c|c|}
\hline System 1 & CASSCF & DMET_1E & DMET & scDMET & LASSCF \\
\hline Singlet (Eh) & -1818.867111 & -1818.866680 & -1818.827114 & -1818.828020 & -1818.866760 \\
\hline Quintet (Eh) & -1818.991333 & -1818.986832 & -1819.114913 & -1818.684053 & -1818.991336 \\
\hline Gap (kcal/mol) & -77.95 & -75.40 & -180.60 & 90.34 & -78.17 \\
\hline
\end{tabular}

Table S2: Singlet and quintet energies (in Hartree) and gaps (in kcal/mol) for $[\mathrm{Fe}(\mathrm{NCH}) 6]+2 \mathrm{with}$ different basis sets and active spaces as calculated by CASSCF, DMET_1E and LASSCF

\begin{tabular}{|c|c|c|c|}
\hline System 1 & CASSCF & DMET_1E & LASSCF \\
\hline$(6,5) 6-31 G-S i n g l e t(E h)$ & -1818.867111 & -1818.866680 & -1818.866759 \\
\hline$(6,5) 6-31 G-Q u i n t e t(E h)$ & -1818.991333 & -1818.986832 & -1818.991336 \\
\hline$(6,5) 6-31 G(d)-S i n g l e t(E h)$ & -1819.121765 & -1819.121298 & -1819.121466 \\
\hline$(6,5) 6-31 G(d)-Q u i n t e t(E h)$ & -1819.237846 & -1819.233030 & -1819.237846 \\
\hline$(6,10) 6-31 G-S i n g l e t(E h)$ & -1818.939077 & -1818.937200 & -1818.938898 \\
\hline$(6,10) 6-31 G-Q u i n t e t(E h)$ & -1819.046836 & -1819.040598 & -1819.046428 \\
\hline$(6,10) 6-31 G(d)-S i n g l e t(E h)$ & -1819.194924 & -1819.191920 & -1819.192141 \\
\hline$(6,10) 6-31 G(d)-Q u i n t e t(E h)$ & -1819.293829 & -1819.286834 & -1819.292020 \\
\hline$(10,12) 6-31 G-S i n g l e t(E h)$ & -1818.961739 & -1818.958590 & -1818.961166 \\
\hline$(10,12) 6-31 G-Q u i n t e t(E h)$ & -1819.052125 & -1819.045726 & -1819.051668 \\
\hline$(10,12) 6-31 G(d)-S i n g l e t(E h)$ & -1819.219486 & -1819.215213 & -1819.219113 \\
\hline$(10,12) 6-31 G(d)-Q u i n t e t(E h)$ & -1819.299113 & -1819.292105 & -1819.297321 \\
\hline$(12,14) 6-31 G(d)-S i n g l e t(E h)$ & -1819.231902 & -1819.227362 & -1819.228015 \\
\hline$(12,14) 6-31 G(d)-Q u i n t e t(E h)$ & -1819.307159 & -1819.299733 & -1819.304990 \\
\hline
\end{tabular}


Table S3: Singlet and quintet energies (in Hartree) and gaps (in $\mathrm{kcal} / \mathrm{mol}$ ) for system $\mathbf{2}$ with a $(6,5)$ active space in a 6-31G* basis using CASSCF, DMET_1E and LASSCF.

\begin{tabular}{|c|c|c|c|}
\hline System 2 & CASSCF & DMET_1E & LASSCF \\
\hline Singlet (Eh) & -2244.420676 & -2244.420260 & -2244.420403 \\
\hline Quintet (Eh) & -2244.520601 & -2244.493554 & -2244.518483 \\
\hline Gap (kcal/mol) & -62.70 & -45.99 & -61.55 \\
\hline
\end{tabular}

Table S4: Singlet and quintet energies (in Hartree) and gaps (in $\mathrm{kcal} / \mathrm{mol}$ ) for system 2 with a $(6,10)$ active space in a 6-31G* basis using CASSCF, DMET_1E and LASSCF.

\begin{tabular}{|c|c|c|c|}
\hline System 2 & CASSCF & DMET_1E & LASSCF \\
\hline Singlet (Eh) & -2244.48708 & -2244.485793 & -2244.486274 \\
\hline Quintet (Eh) & -2244.582641 & -2244.571958 & -2244.577132 \\
\hline Gap (kcal/mol) & -59.97 & -54.07 & -57.01 \\
\hline
\end{tabular}

Table S5 : The absolute energies (in Hartree) for system 3 calculated using 6-31G* basis set and a $(6,5)$ on each iron for LASSCF and $(12,10)$ active space for the CASSCF

\begin{tabular}{|c|c|c|c|c|}
\hline Overall Spin & $\mathrm{E}_{\mathrm{CASSCF}}$ & $m_{s}$ for Fe1 (LAS) & $m_{s}$ for $\mathrm{Fe} 2(\mathrm{LAS})$ & E $_{\text {LASSCF }}$ \\
\hline \multirow{3}{*}{ Singlet } & \multirow{3}{*}{-3655.999778} & 0 & 0 & -3655.793325 \\
\hline & & 1 & -1 & -3655.841406 \\
\hline & & 2 & -2 & -3655.997415 \\
\hline Nonet & -3655.999764 & 2 & 2 & -3655.997411 \\
\hline
\end{tabular}

We also note that if the geometry is optimized for an overall singlet (using the same computational methods), and then used to study the coupling, we get a similar behavior i.e the anti-ferromagnetic quintets are more stable and closer to CASSCF than the diamagnetic singlets. The energies (table S6) are significantly different than the ones in table S5 mainly because of the change in the geometry of the molecule.

Table S6 : The absolute energies (in Hartree) for singlet geometry of system 3 calculated using 6-31G* basis set and a $(6,5)$ on each iron for LASSCF and $(12,10)$ active space for the CASSCF 


\begin{tabular}{|c|c|c|c|c|}
\hline Overall Spin & E(CASSCF) & $m_{s}$ for Fe1 (LAS) & $m_{s}$ for Fe1 (LAS) & E(LASSCF) \\
\hline \multirow{2}{*}{ Singlet } & \multirow{2}{*}{-3655.914770} & 2 & -2 & -3655.903912 \\
\cline { 3 - 5 } & & 0 & 0 & -3655.777383 \\
\hline
\end{tabular}

\section{Geometries Optimized at PBE/Def2TZVP in Gaussian 09.}

Reported in Angstroms

System 1-Singlet:

$E=-1823.17743134$ Hartree

$\begin{array}{llll}\mathrm{Fe} & 0.000000000 & 0.000000000 & 0.000000000\end{array}$

$\begin{array}{llll}\mathrm{N} & 0.000000000 & 0.000000000 & 1.943469000\end{array}$

$\begin{array}{llll}\mathrm{N} & 0.000000000 & 1.943469000 & 0.000000000\end{array}$

$\begin{array}{llll}\mathrm{N} & -1.943469000 & 0.0000000000 & 0.000000000\end{array}$

$\mathrm{N} \quad 0.000000000 \quad-1.943469000 \quad 0.000000000$

$\mathrm{N} \quad 0.000000000 \quad 0.000000000 \quad-1.943469000$

$\mathrm{N} \quad 1.943469000 \quad 0.000000000 \quad 0.000000000$

$\begin{array}{llll}\text { C } & 0.000000000 & 0.000000000 & 3.082693000\end{array}$

$\mathrm{H} \quad 0.000000000 \quad 0.000000000 \quad 4.157700000$

$\begin{array}{llll}\text { C } & -3.082693000 & 0.000000000 & 0.000000000\end{array}$

$\mathrm{H} \quad-4.157700000 \quad 0.000000000 \quad 0.000000000$

$\begin{array}{llll}\text { C } & 0.000000000 & -3.082693000 & 0.000000000\end{array}$

$\mathrm{H} \quad 0.000000000 \quad-4.157700000 \quad 0.000000000$

$\begin{array}{llll}\text { C } & 0.000000000 & 3.082693000 & 0.000000000\end{array}$

$\begin{array}{llll}\mathrm{H} & 0.000000000 & 4.157700000 & 0.000000000\end{array}$

$\begin{array}{llll}\text { C } & 3.082693000 & 0.000000000 & 0.000000000\end{array}$

$\begin{array}{llll}\mathrm{H} & 4.157700000 & 0.000000000 & 0.000000000\end{array}$

$\begin{array}{llll}\text { C } & 0.000000000 & 0.000000000 & -3.082693000\end{array}$

$\mathrm{H} \quad 0.000000000 \quad 0.000000000 \quad-4.157700000$ 


\section{System 1-Quintet:}
$\mathrm{E}=-1823.19089875$ Hartree
Fe $\quad 0.000000000 \quad 0.000000000 \quad 0.000000000$
$\begin{array}{llll}\mathrm{N} & 0.000000000 & 2.174859000 & 0.000000000\end{array}$
$\begin{array}{llll}\mathrm{N} & -2.174859000 & 0.000000000 & 0.000000000\end{array}$
$\begin{array}{llll}\mathrm{N} & 0.000000000 & 0.000000000 & 2.184759000\end{array}$
$\begin{array}{llll}\mathrm{N} & 2.174859000 & 0.000000000 & 0.000000000\end{array}$
$\begin{array}{llll}\mathrm{N} & 0.000000000 & -2.174859000 & 0.000000000\end{array}$
$\begin{array}{llll}\mathrm{N} & 0.000000000 & 0.000000000 & -2.184759000\end{array}$
$\begin{array}{llll}\text { C } & 0.000000000 & 3.315742000 & 0.000000000\end{array}$
$\begin{array}{llll}\mathrm{H} & 0.000000000 & 4.390934000 & 0.000000000\end{array}$
$\begin{array}{llll}\text { C } & 0.000000000 & 0.000000000 & 3.325070000\end{array}$
$\begin{array}{llll}\mathrm{H} & 0.000000000 & 0.000000000 & 4.400783000\end{array}$
$\begin{array}{llll}\text { C } & 3.315742000 & 0.000000000 & 0.000000000\end{array}$
$\begin{array}{llll}\mathrm{H} & 4.390934000 & 0.000000000 & 0.000000000\end{array}$
$\begin{array}{llll}\text { C } & -3.315742000 & 0.000000000 & 0.000000000\end{array}$
$\begin{array}{llll}\mathrm{H} & -4.390934000 & 0.000000000 & 0.000000000\end{array}$
$\begin{array}{llll}\text { C } & 0.000000000 & 0.000000000 & -3.325070000\end{array}$
H $\quad 0.000000000 \quad 0.000000000 \quad-4.400783000$
$\begin{array}{llll}\text { C } & 0.000000000 & -3.315742000 & 0.000000000\end{array}$
$\mathrm{H} \quad 0.000000000 \quad-4.390934000 \quad 0.000000000$

\section{System 2-Singlet:}

$E=-2251.11318343$ Hartree

Fe $\quad-0.000001000 \quad-0.000001000 \quad-0.000117000$

$\begin{array}{llll}\text { C } & 1.213286000 & -2.764891000 & -0.000022000\end{array}$

$\mathrm{N} \quad 1.401826000 \quad-1.412035000 \quad-0.000057000$

$\begin{array}{llll}\text { C } & 2.756011000 & -1.233317000 & -0.000004000\end{array}$ 


\begin{tabular}{lrrr}
$\mathrm{C}$ & -2.497804000 & -3.432657000 & 0.000224000 \\
$\mathrm{C}$ & -3.450695000 & -2.472829000 & -0.000018000 \\
$\mathrm{C}$ & -2.764892000 & -1.213285000 & 0.000084000 \\
$\mathrm{~N}$ & -1.412037000 & -1.401823000 & -0.000031000 \\
$\mathrm{C}$ & -1.233318000 & -2.756009000 & 0.000028000 \\
$\mathrm{C}$ & 2.497806000 & 3.432658000 & -0.000225000 \\
$\mathrm{C}$ & 3.450696000 & 2.472831000 & 0.000154000 \\
$\mathrm{C}$ & 2.764893000 & 1.213285000 & -0.000021000 \\
$\mathrm{~N}$ & 1.412038000 & 1.401824000 & -0.000034000 \\
$\mathrm{C}$ & 1.233318000 & 2.756010000 & 0.000013000 \\
$\mathrm{C}$ & -3.432659000 & 2.497807000 & -0.000032000 \\
$\mathrm{C}$ & -2.472828000 & 3.450694000 & 0.000116000 \\
$\mathrm{C}$ & -1.213285000 & 2.764887000 & 0.000044000 \\
$\mathrm{~N}$ & -1.401827000 & 1.412034000 & -0.000014000 \\
$\mathrm{C}$ & -2.756015000 & 1.233318000 & 0.000060000 \\
$\mathrm{C}$ & 3.432659000 & -2.497804000 & 0.000023000 \\
$\mathrm{C}$ & 2.472831000 & -3.450694000 & -0.000030000 \\
$\mathrm{C}$ & -0.012332000 & -3.398723000 & 0.000013000 \\
$\mathrm{C}$ & 0.012333000 & 3.398722000 & 0.000046000 \\
$\mathrm{C}$ & -3.398727000 & 0.012333000 & 0.000095000 \\
$\mathrm{C}$ & 3.398726000 & -0.012332000 & 0.000013000 \\
$\mathrm{H}$ & -2.621222000 & -4.505682000 & 0.000360000 \\
$\mathrm{H}$ & -4.524586000 & -2.588461000 & -0.000069000 \\
$\mathrm{H}$ & 2.621222000 & 4.505683000 & -0.000383000 \\
$\mathrm{H}$ & 4.524588000 & 2.588460000 & 0.000297000 \\
$\mathrm{H}$ & -4.505683000 & 2.621227000 & -0.000089000 \\
\hline & -2.588455000 & 4.524586000 & 0.000185000 \\
\hline & -0.516267000 & -4.482027000 & 0.000062000
\end{tabular}




$\begin{array}{rrrr}\mathrm{H} & 0.016263000 & 4.482027000 & 0.000042000 \\ \mathrm{H} & -4.482032000 & 0.016263000 & 0.000090000 \\ \mathrm{H} & 4.482031000 & -0.016267000 & 0.000088000\end{array}$

\section{System 2 - Quintet:}

$\begin{array}{lrrr}\mathrm{E}= & -2251.15670042 & \text { Hartree } & \\ \mathrm{Fe} & 0.000000000 & 0.000000000 & 0.000008000 \\ \mathrm{C} & -1.220852000 & -2.808145000 & -0.000020000 \\ \mathrm{~N} & -1.437594000 & -1.463982000 & -0.000034000 \\ \mathrm{C} & -2.785474000 & -1.271683000 & -0.000037000 \\ \mathrm{C} & 2.545340000 & -3.451185000 & -0.000018000 \\ \mathrm{C} & 3.496877000 & -2.482200000 & -0.000108000 \\ \mathrm{C} & 2.808148000 & -1.220851000 & 0.000012000 \\ \mathrm{~N} & 1.463985000 & -1.437591000 & 0.000043000 \\ \mathrm{C} & 1.271684000 & -2.785471000 & -0.000014000 \\ \mathrm{C} & -2.545340000 & 3.451185000 & -0.000005000 \\ \mathrm{C} & -3.496877000 & 2.482201000 & 0.000119000 \\ \mathrm{C} & -2.808148000 & 1.220851000 & -0.000019000 \\ \mathrm{~N} & -1.463985000 & 1.437592000 & -0.000042000 \\ \mathrm{C} & -1.271684000 & 2.785472000 & 0.000013000 \\ \mathrm{C} & 3.451186000 & 2.545341000 & -0.000012000 \\ \mathrm{C} & 2.482199000 & 3.496876000 & 0.000037000 \\ \mathrm{C} & 1.220852000 & 2.808143000 & 0.000020000 \\ \mathrm{~N} & 1.437595000 & 1.463981000 & 0.000034000 \\ \mathrm{C} & 2.785476000 & 1.271684000 & 0.000034000 \\ \mathrm{C} & -3.451186000 & -2.545340000 & -0.000006000 \\ \mathrm{C} & -2.482201000 & -3.496876000 & -0.000015000 \\ \mathrm{C} & 0.031053000 & -3.416769000 & -0.000018000 \\ \mathrm{C} & -0.031053000 & 3.416768000 & 0.000017000\end{array}$




$\begin{array}{lrrr}\mathrm{C} & 3.416773000 & 0.031053000 & 0.000027000 \\ \mathrm{C} & -3.416773000 & -0.031053000 & -0.000035000 \\ \mathrm{H} & 2.687770000 & -4.522157000 & -0.000026000 \\ \mathrm{H} & 4.570262000 & -2.605144000 & -0.000182000 \\ \mathrm{H} & -2.687770000 & 4.522157000 & -0.000012000 \\ \mathrm{H} & -4.570263000 & 2.605144000 & 0.000204000 \\ \mathrm{H} & 4.522158000 & 2.687774000 & -0.000037000 \\ \mathrm{H} & 2.605140000 & 4.570262000 & 0.000043000 \\ \mathrm{H} & -4.522158000 & -2.687771000 & 0.000005000 \\ \mathrm{H} & -2.605142000 & -4.570262000 & -0.000002000 \\ \mathrm{H} & 0.040926000 & -4.500978000 & -0.000018000 \\ \mathrm{H} & -0.040924000 & 4.500977000 & 0.000019000 \\ \mathrm{H} & 4.500983000 & 0.040924000 & -0.000004000 \\ \mathrm{H} & -4.500982000 & -0.040926000 & -0.000007000\end{array}$

\section{System 3 -Nonet:}

$E=-3663.96388223$

$\begin{array}{llll}\mathrm{Fe} & -3.004847000 & 0.000000000 & 0.000000000\end{array}$

$\begin{array}{llll}\mathrm{Fe} & 3.004847000 & 0.000000000 & 0.000000000\end{array}$

$\begin{array}{llll}\text { C } & 0.000000000 & 3.409725000 & 0.000000000\end{array}$

$\begin{array}{llll}\text { C } & -1.181171000 & 2.688098000 & 0.000000000\end{array}$

$\begin{array}{llll}\text { C } & 0.000000000 & 0.740752000 & 0.000000000\end{array}$

$\begin{array}{llll}\text { C } & 1.181171000 & 2.688098000 & 0.000000000\end{array}$

$\begin{array}{llll}\text { C } & 0.000000000 & -0.740752000 & 0.000000000\end{array}$

$\begin{array}{llll}\text { C } & -1.181171000 & -2.688098000 & 0.000000000\end{array}$

$\mathrm{H} \quad-2.146819000 \quad-3.183003000 \quad 0.000000000$

$\begin{array}{llll}\text { C } & 0.000000000 & -3.409725000 & 0.000000000\end{array}$

$\begin{array}{llll}\text { C } & 1.181171000 & -2.688098000 & 0.000000000\end{array}$

$\begin{array}{llll}\mathrm{H} & 0.000000000 & 4.493448000 & 0.000000000\end{array}$ 


\begin{tabular}{|c|c|c|c|}
\hline $\mathrm{H}$ & -2.146819000 & 3.183003000 & 0.000000000 \\
\hline$H$ & 2.146819000 & 3.183003000 & 0.000000000 \\
\hline $\mathrm{H}$ & 0.000000000 & -4.493448000 & 0.000000000 \\
\hline $\mathrm{H}$ & 2.146819000 & -3.183003000 & 0.000000000 \\
\hline $\mathrm{N}$ & 1.181856000 & -1.349331000 & 0.000000000 \\
\hline $\mathrm{N}$ & 1.181856000 & 1.349331000 & 0.000000000 \\
\hline $\mathrm{N}$ & -1.181856000 & 1.349331000 & 0.000000000 \\
\hline $\mathrm{N}$ & -1.181856000 & -1.349331000 & 0.000000000 \\
\hline $\mathrm{O}$ & -3.144689000 & 0.000000000 & 2.173794000 \\
\hline $\mathrm{H}$ & -3.215015000 & 0.767135000 & 2.755999000 \\
\hline $\mathrm{H}$ & -3.215015000 & -0.767135000 & 2.755999000 \\
\hline $\mathrm{O}$ & -4.456206000 & -1.575287000 & 0.000000000 \\
\hline $\mathrm{H}$ & -4.991908000 & -1.818728000 & -0.767267000 \\
\hline $\mathrm{H}$ & -4.991908000 & -1.818728000 & 0.767267000 \\
\hline $\mathrm{O}$ & -4.456206000 & 1.575287000 & 0.000000000 \\
\hline $\mathrm{H}$ & -4.991908000 & 1.818728000 & -0.767267000 \\
\hline $\mathrm{H}$ & -4.991908000 & 1.818728000 & 0.767267000 \\
\hline $\mathrm{O}$ & 4.456206000 & -1.575287000 & 0.000000000 \\
\hline $\mathrm{H}$ & 4.991908000 & -1.818728000 & -0.767267000 \\
\hline $\mathrm{H}$ & 4.991908000 & -1.818728000 & 0.767267000 \\
\hline $\mathrm{O}$ & 4.456206000 & 1.575287000 & 0.000000000 \\
\hline $\mathrm{H}$ & 4.991908000 & 1.818728000 & -0.767267000 \\
\hline $\mathrm{H}$ & 4.991908000 & 1.818728000 & 0.767267000 \\
\hline $\mathrm{O}$ & 3.144689000 & 0.000000000 & 2.173794000 \\
\hline $\mathrm{H}$ & 3.215015000 & -0.767135000 & 2.755999000 \\
\hline $\mathrm{H}$ & 3.215015000 & 0.767135000 & 2.755999000 \\
\hline $\mathrm{O}$ & -3.144689000 & 0.000000000 & -2.173794000 \\
\hline $\mathrm{H}$ & -3.215015000 & 0.767135000 & -2.755999000 \\
\hline $\mathrm{H}$ & -3.215015000 & -0.767135000 & -2.755999000 \\
\hline $\mathrm{O}$ & 3.144689000 & 0.000000000 & -2.173794000 \\
\hline
\end{tabular}




$\begin{array}{llll}\mathrm{H} & 3.215015000 & -0.767135000 & -2.755999000 \\ \mathrm{H} & 3.215015000 & 0.767135000 & -2.755999000\end{array}$

References:

(1) Frisch; Trucks, G.; Schlegel, H.; Scuseria, G.; Robb, M.; Cheeseman, J.; Scalmani, G.; Barone, V.; Mennucci, B.; Petersson, G.; et al. Gaussian 09, Revision B.01. Gaussian 09, Revis. B.01, Gaussian, Inc., Wallingford CT 2009.

(2) Perdew, J. P.; Burke, K.; Ernzerhof, M. Generalized Gradient Approximation Made Simple. Phys. Rev. Lett. 1996, 77 (18), 3865.

(3) Weigend, F.; Ahlrichs, R. Balanced Basis Sets of Split Valence, Triple Zeta Valence and Quadruple Zeta Valence Quality for H to Rn: Design and Assessment of Accuracy. Phys. Chem. Chem. Phys. 2005, 7 (18), 3297. https://doi.org/10.1039/b508541a.

(4) Sun, Q.; Chan, G. K.-L. Exact and Optimal Quantum Mechanics/Molecular Mechanics Boundaries. J. Chem. Theory Comput. 2014, 10 (9), 3784-3790.

(5) Sun, Q.; Berkelbach, T. C.; Blunt, N. S.; Booth, G. H.; Guo, S.; Li, Z.; Liu, J.; McClain, J. D.; Sayfutyarova, E. R.; Sharma, S.; et al. PySCF: The Python-based Simulations of Chemistry Framework. Wiley Interdisciplinary Reviews: Computational Molecular Science. 2017, p e1340. https://doi.org/10.1002/wcms.1340.

(6) Sayfutyarova, E. R.; Sun, Q.; Chan, G. K.-L.; Knizia, G. Automated Construction of Molecular Active Spaces from Atomic Valence Orbitals. J. Chem. Theory Comput. 2017, 13 (9), 4063-4078. https://doi.org/10.1021/acs.jctc.7b00128. 
